\title{
Longitudinal Associations Between Sexual Communication With Friends and Sexual Behaviors Through Perceived Sexual Peer Norms
}

Raquel Nogueira Avelar e Silva, Hein Raat, Ellen Reitz, Merel Plat, Maja Deković \& Daphne Van De Bongardt

To cite this article: Raquel Nogueira Avelar e Silva, Hein Raat, Ellen Reitz, Merel Plat, Maja Deković \& Daphne Van De Bongardt (2019): Longitudinal Associations Between Sexual Communication With Friends and Sexual Behaviors Through Perceived Sexual Peer Norms, The Journal of Sex Research, DOI: 10.1080/00224499.2019.1691969

To link to this article: https://doi.org/10.1080/00224499.2019.1691969

册 Published online: 21 Nov 2019.

Submit your article to this journal ๔

Џ Article views: 56

Q View related articles

View Crossmark data $\nearrow$ 


\title{
Longitudinal Associations Between Sexual Communication With Friends and Sexual Behaviors Through Perceived Sexual Peer Norms
}

\author{
Raquel Nogueira Avelar e Silva (10) ${ }^{a}$, Hein Raat ${ }^{a}$, Ellen Reitz ${ }^{b}$, Merel Plat ${ }^{c}$, Maja Dekovićb, and Daphne Van De Bongardt ${ }^{d}$
}

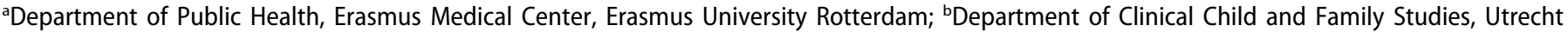

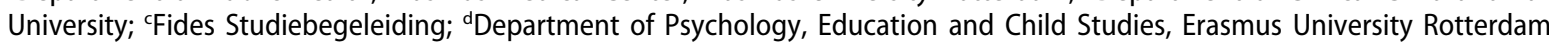

\begin{abstract}
The role of peers in adolescents' sexual behaviors is not yet fully understood. We investigated the association between sexual communication with friends (at T1) and subsequent changes in adolescents' experience with sexual behaviors (between T1-T3), and examined whether this association was explained by adolescents' perceptions of three sexual peer norms (at T2): (1) peers' sexual behaviors (descriptive norms), (2) peers' approval of sexual behaviors (injunctive norms), and (3) peer pressure to have sex. The data source was Project STARS, a longitudinal study on adolescent sexual development in the Netherlands, collected via online self-report questionnaires from 1,116 adolescents (11.5-17.9 years). Adolescents who communicated more frequently with their friends about sexuality-related topics at T1 reported significantly larger increases in their experience with different sexual behaviors between T1-T3. More sexual communication with friends also predicted adolescents subsequently perceiving more 1) peer sexual behaviors, 2) peer approval of sex, and 3) peer pressure to have sex. These stronger perceptions, in turn, predicted larger increases in their sexual behaviors between T1-T3. After adjusting for the three norms simultaneously, the main association between sexual communication with friends and sexual behavior change weakened but remained significant. Inspection of specific indirect effects showed this link was explained by injunctive norms only. No gender differences were found.
\end{abstract}

The initiation of intimate relationships and sexual behaviors is a normative part of adolescent development (Van de Bongardt, De Graaf, Reitz, \& Deković, 2014). According to the ecological approach, which has become a dominant paradigm in research on adolescent sexuality, different factors (e.g., individual, social) play a role in adolescent sexual development (Smetana, Campione-Barr, \& Metzger, 2006). Different ecological systems theories, for example, emphasize that both individual factors (such as age, gender, ethnic background) and social factors (such as parents, peers) can affect adolescent sexual behaviors (Bronfenbrenner, 1994). Parents are important protective factors of adolescent sexual health (Nogueira Avelar E Silva, Van de Bongardt, Van de Looijjansen, Wijtzes, \& Raat, 2016; VVan de Bongardt et al., 2014). However, particularly during adolescence, peers (e.g., friends, classmates, age-mates) become increasingly present in adolescents' social contexts. As a result, they become a notable reference group for adolescents' behavioral decisions, including decisions about sexual behaviors (Steinberg \& Morris, 2001). Thus, the role of peers in adolescents' sexual behaviors is critical to consider in research on adolescent sexuality (Berten \& Van Rossem, 2011; Mollborn \& Sennott, 2015; Sennott \& Mollborn, 2011; Warner, 2018).

Previous empirical research that has investigated peer aspects in association with adolescents' sexual behaviors has shown, for instance, that more frequent communication with friends about sexuality-related topics was associated with a higher likelihood of subsequent sexual intercourse initiation (Busse, Fishbein, Bleakley, \& Hennessy, 2010). A possible explanation for this association may be that, during sexual talks, friends exchange information about their sexual behaviors, which can contribute to increasing adolescents' awareness of their friends' sexual activity. In turn, this increase in levels of adolescents' perceptions of their friends' sexual behaviors could stimulate adolescents' sexual behaviors through role modeling (Bandura, 1971).

These mechanisms by which perceptions of a group of people (e.g., peers) can contribute to shaping others' behavior (e.g., adolescents' sexual behaviors) have long been described in the literature in terms of social norms (Bicchieri, 2006, 2017; Cialdini \& Goldstein, 2004). Social norm theory posits that individuals, in general, tend to behave according to social norms that they perceive as prevalent, accepted, and desired among relevant social agents, such as their peers (Bicchieri, 2006, 2017; Cialdini \& Goldstein, 2004). At the core of social norm theory lies the notion that people do not make choices in isolation; instead, they pay attention to (their expectations of) how other people behave, what they approve or disapprove, and what they consider appropriate (Bicchieri, 2017). Thus, adolescents would be more likely to engage in sexual behaviors when they perceive those behaviors as usual among their peers (Van de Bongardt, Reitz, Sandfort, \& Deković,

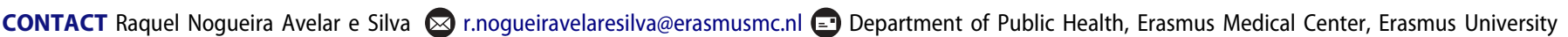
Rotterdam, P.O. Box 2040, Rotterdam, CA P.O. Box 2040, The Netherlands

(c) 2019 The Society for the Scientific Study of Sexuality 
2015). Understanding how social norms (e.g., sexual norms among peers) affect behaviors (e.g., sexual behaviors) is critical to the promotion of behavioral change toward adolescents adopting healthy behaviors, such as the timing of engagement in different sexual behaviors (Bicchieri, 2017). For example, if public health policies aimed at improving adolescents' sexual health, we need to have an in-depth understanding of how peers play a role in their sexual development, including their engagement in sexual behaviors (Mollborn \& Sennott, 2015; Sennott \& Mollborn, 2011).

The literature has conceptually distinguished the three types of sexual peer norms: Descriptive norms, injunctive norms, and more explicit peer pressure (Van de Bongardt et al., 2015). Descriptive norms refer to adolescents' perceptions of their peers' sexual behaviors (e.g., adolescents' perceptions of how many of their friends have ever had sexual intercourse; Ali \& Dwyer, 2011; East, Khoo, \& Reyes, 2006; Fasula \& Miller, 2006; Van de Bongardt et al., 2014). These norms (i.e., what peers $d o$ ) could motivate adolescents' sexual behaviors because they might reason that if their friends behave in a certain way, it must be a good or wise thing to do (Ali \& Dwyer, 2011). Injunctive norms refer to adolescents' perceptions of their peers' (dis)approval toward sex (i.e., what peers think; Akers et al., 2011; East et al., 2006). Several studies showed that adolescents who perceived that their friends were more permissive toward sex were more likely to engage in sexual behaviors themselves (O'Sullivan \& BrooksGunn, 2005; Santelli et al., 2004). Peer pressure refers to an explicit social pressure that adolescents perceive from their peers to engage in sexual behaviors, regardless of adolescents' own wishes (East et al., 2006; Santor, Messervey, \& Kusumakar, 2000). Adolescents may be motivated to conform to such peer pressure (e.g., to engage in the expected behavior) because of perceived social benefits (e.g., social acceptance, or an increase in popularity) when they do conform, or social losses (e.g., social rejection, or a decrease in popularity) when they do not conform. A metaanalysis has shown that these three kinds of sexual peer norms have a unique predictive value in relation to adolescents' own sexual behaviors (Van de Bongardt et al., 2015). More specifically, the strength of the associations differ: Descriptive norms were most strongly related to adolescents' own sexual behaviors (i.e., adolescents who believed that more of their friends were sexually experienced, were more likely to engage in sexual behaviors themselves), whereas peer pressure showed the weakest link with adolescents' own sexual behaviors (Van de Bongardt et al., 2015).

Although adolescents' perceptions of sexual peer norms may partly explain the identified link between sexual communication with friends and adolescents' sexual behaviors, only one longitudinal study has investigated these interlinkages (Busse et al., 2010). This study was conducted in the United States and included 14-16-year old adolescents (Busse et al., 2010). Thus, the role of sexual peer norms as an explanatory mechanism in the link between sexual communication with friends and sexual behaviors is not yet fully established in the literature. Also, we do not know whether the findings from the American study are generalizable to adolescents from other countries.

Besides assessing the association between sexual communication with friends and sexual intercourse initiation, the study of Busse et al. (2010) examined whether this association was explained by two types of sexual peer norms: Descriptive and injunctive norms, which, for analysis, were combined into a new variable: "normative pressure", based on the average scores on descriptive and injunctive norms. The results of the mediation analysis showed that adolescents who communicated more frequently with their friends about sexuality-related topics perceived more of this normative pressure, that is: that their friends had increased experience with sexual behaviors (descriptive norm) and that their friends approved more of having sex (injunctive norm). Subsequently, these combined perceptions were associated with a higher likelihood of sexual intercourse initiation (Busse et al., 2010). While this study contributed to advance our understanding of how friends may play a role in adolescents' sexual behaviors through sexual communication and sexual norms, the present study aimed to further advance this understanding through three additional methodological considerations.

First, Busse et al. (2010) examined two types of sexual peer norms only. Theoretically and empirically, all three types of norms (i.e., descriptive norms, injunctive norms, and peer pressure) are relevant to consider in research on adolescent sexual development because they are distinct and have different predictive values (Van de Bongardt et al., 2015). Additionally, although Busse et al. (2010) measured descriptive and injunctive norms separately, for analysis, they were combined (averaged) into one social norm variable "normative pressure". The use of such a composite variable is not conceptually consistent with social norm theory, nor with empirical research that shows that all three types of sexual peer norms are distinct and have a unique predictive value (Cialdini \& Goldstein, 2004; Van de Bongardt et al., 2015). In the current study, therefore, we included all three types of sexual peer norms (i.e., descriptive norms, injunctive norms, and peer pressure), and assessed their unique explanatory role (i.e., mediating effect) in the link between sexual communication with friends and adolescents' sexual behaviors.

Second, the study of Busse et al. (2010) included exclusively sexual intercourse behavior. This inclusion is a rather narrow conceptualization of sexual behavior, considering that most adolescents progress from less intimate sexual behaviors (e.g., naked touching) to increasingly more intimate behaviors (e.g., sexual intercourse; De Graaf, Van de Borne, \& Meijer, 2018; De Graaf, Vanwesenbeeck, Meijer, Woertman, \& Meeus, 2009). Hence, as a considerable part $( \pm 75 \%)$ of adolescents engage in other non-coital sexual behaviors before their first sexual intercourse experience, a sole focus on sexual intercourse provides rather limited insight into adolescents' sexual activity. Our study, therefore, extends the current literature by utilizing a broader conceptualization of sexual behaviors, which varied from naked touching to sexual intercourse, and thus more accurately reflects the reality of adolescents' developing sexuality and the role of peers therein.

Third, although the study of Busse et al. (2010) examined sexual communication with friends in real life, in contemporary societies, adolescents are increasingly using online communication, such as instant messaging, to communicate with their peers on a daily basis (Doornwaard, Moreno, Van den Eijnden, Vanwesenbeeck, \& Ter Bogt, 2014; Doornwaard, Van den Eijnden, Overbeek, \& Ter Bogt, 2015). Moreover, the majority of adolescents tend to share personal information (thoughts, feelings, and experiences) almost 
as frequently during online communication with their friends as during face-to-face communication (Valkenburg, Sumter, \& Peter, 2011). To the authors' knowledge, no studies have yet included both types of sexual communication with friends in the investigation of the interlinkages between communication about sex with friends and their sexual behaviors. Our study extends the literature by including both types of communication with friends (i.e., online and face-to-face).

In sum, in the present longitudinal study, we assessed the association between sexual communication (online and face-toface) with friends at Time 1 (T1) and changes in adolescents' experiences with different sexual behaviors (i.e., ranging from naked touching to intercourse) over time, between Time 1 and 3 (T1-T3). In addition, we examined whether this association was mediated (i.e., explained) by three types of sexual peer norms at Time 2 (T2): Descriptive norms, injunctive norms, and peer pressure. Based on the aforementioned theoretical rationale and empirical evidence, firstly, we hypothesized that adolescents who communicated more frequently (online and face-to-face) with their friends about sexuality-related topics at T1 would gain experience with more types of sexual behaviors between T1-T3 (H1). Secondly, we hypothesized that adolescents who communicated more frequently (online and face-to-face) with their friends about sexuality-related topics at $\mathrm{T} 1$ would perceive, at T2, that: 1) more of their friends had experience with sexual behaviors (i.e., descriptive norms), 2) their friends approved more of having sex (i.e., injunctive norms), and 3) there was more pressure from their peers to have sex (H2). Thirdly, we hypothesized that adolescents who perceived, at T2, that: 1) more of their friends had experiences with sexual behaviors, 2) their friends approved more of having sex, and 3) their peers exerted more pressure to have sex would over time gain experience with more types of sexual behaviors between T1-T3 (H3). Fourthly, we hypothesized that the association between sexual communication with friends at $\mathrm{T} 1$ and adolescents' increases in sexual behaviors between T1-T3 would be mediated by all three types of sexual peer norms at T2. Specifically, we expected that the mediating effect of descriptive norms would be stronger than those of injunctive norms and peer pressure (H4; Van de Bongardt et al., 2015).

Finally, we examined gender differences in both direct and indirect associations between sexual communication with friends and changes in sexual behaviors through sexual peer norms. In general, gender differences are expected in research on adolescent sexuality. This may be related to the culture of sexual double standards that exist in many societies, meaning that the expectations for boys' and girls' sexual behaviors are different (Kreager \& Staff, 2009). Usually, boys are granted more sexual freedom, and girls tend to experience more sexual restrictions (Kreager \& Staff, 2009). These cultural differences in expectations for boys' and girls' sexual behaviors often translate into more positive evaluations of boys' and more negative evaluations of girls' (early) sexual activity (Kreager \& Staff, 2009). Accordingly, research has demonstrated gender differences in adolescents' sexual behaviors and the interlinkages between sexuality-related peer aspects and sexual behaviors (Kapungu et al., 2010; Van de Bongardt et al., 2015). Moreover, overall, girls tend to be more susceptible to social influences (Cialdini \& Goldstein, 2004) and tend to be more sensitive to peers' social evaluations than boys (Rudolph \& Conley, 2005). Thus, our final hypothesis was that both direct and indirect associations between sexual communication with friends and sexual behaviors through perceived sexual peer norms would be stronger for girls than for boys (H5).

\section{Method}

\section{Participants}

Data for the current study were collected as part of Project STARS (Studies on Trajectories of Adolescent Relationships and Sexuality), a large-scale longitudinal study on adolescent sexual development, conducted in the Netherlands between 2010 and 2015 (Reitz et al., 2015). Longitudinal questionnaire data were collected among a school-based sample of 1,297 10to-19-year-old adolescents, with 6-month intervals between measurements $(\mathrm{T} 1=$ Fall 2011, $\mathrm{T} 2=$ Spring $2012, \mathrm{~T} 3=$ Fall 2012, T4 = Spring 2013). Participants were recruited from four secondary and eight elementary schools throughout the Netherlands. Adolescents and their parents received letters, brochures, and flyers, describing the aims of the study. Parents received a form on which they could indicate if they did not want their child to participate in the study (i.e., passive informed consent). Less than $7.0 \%$ of the approached adolescents decided not to participate or were not allowed to partake in the study by their parents. Researchers supervised data collection in order to introduce the study and the procedures, answer questions, and ensure privacy. Adolescents completed online questionnaires in the classroom voluntarily. Confidentiality of the responses was guaranteed, as was the option to withdraw participation at any time. After participation, adolescents received a book gift certificate (increasing in value from $€ 5,00$ at $\mathrm{T} 1$ to $€ 12,50$ at T4). Project STARS was approved by the ethics board of Utrecht University in the Netherlands.

\section{Study Sample}

For the current study, we used the first three waves of data. We selected only secondary school students $(n=1,132)$, as the questionnaire for elementary school students $(n=165)$ did not include all investigated instruments. Moreover, we corrected $(n=28)$ or excluded $(n=16)$ over-time inconsistencies in adolescents' reports of their sexual behaviors (e.g., reporting sexual experience at $\mathrm{T} 2$ and $\mathrm{T} 4$, but reporting no sexual experience at $\mathrm{T} 1$ and $\mathrm{T} 3$ ), depending on the available information on sexual behaviors across the waves. This resulted in a final prospective analysis sample of 1,116 adolescents.

\section{Measures}

\section{Sexual Behaviors}

To assess adolescents' experience with non-coital and coital sexual behaviors across time, we used an instrument previously applied in studies using Project STARS data, which has shown good reliability (Doornwaard et al., 2015; Nogueira Avelar E Silva, Van de Bongardt, Baams, \& Raat, 2018; Van de Bongardt et al., 2014). Participants were asked: "Have you 
ever had sex with another person? By sex, we mean everything from touching and caressing to intercourse" ( $0=$ no, $1=$ yes $)$. Participants who answered "yes" subsequently reported on their experience with four types of non-coital and coital sexual behaviors: 1$)$ naked touching or caressing ( $0=$ no, $1=$ yes $), 2)$ manual sex $(0=$ no, 1 = yes $), 3)$ oral sex $(0=$ no, $1=$ yes $)$, and 4$)$ vaginal intercourse $(0=$ no, $1=$ yes $)$. The scores on the four items were summed into one count variable, indicating the level of adolescents' experience with these four behaviors ( 0 = experience with no sexual behavior, $4=$ experience with all four sexual behaviors; Doornwaard et al., 2015; Nogueira Avelar E Silva et al., 2018; Van de Bongardt et al., 2014. Each behavior was scored equally (1) to avoid attributing a different normative meaning to each of them, of which we cannot be sure. Cronbach's alphas, which measure the internal consistency between the items that are used to construct a variable (Rothman, Greenland, \& Lash, 2008), were .85 at T1 and .91 at T3.

\section{Sexual Communication with Friends}

Communication with friends about sexuality was measured with a four-item instrument previously used in a representative Dutch study on young people's sexual health "Sex under the age of 25 " (De Graaf et al., 2018), and other studies (Van de Bongardt et al., 2014). At T1, adolescents were asked, "How often do you discuss the following topics with your friends: 1. Being in love and relationships, 2. What you do or do not want to do sexually; 3 . With whom you do or do not want to have sex and why, and 4. Safe sex: STIs, unwanted pregnancy, condoms, and contraceptives" ( $1=$ never, 6 = very often). They answered these questions twice: once for face-to-face communication ("How often do you discuss these topics with your friends when you are together?"), and another time for online communication ("How often do you discuss these topics with your friends on the Internet, for example through MSN, e-mail, Skype, or chat?". An overall score was calculated by averaging the scores of the eight items $(\alpha=.85)$. Higher scale scores indicated more frequent face-to-face and online communication about sex with friends.

\section{Sexual Peer Norms}

To measure descriptive norms, adolescents' perceptions of their friends' sexual behaviors were assessed at T2 with an item that is often used in the literature (East et al., 2006; Fasula \& Miller, 2006; Van de Bongardt et al., 2014, 2015): "How many of your best friends do you think have experience with intercourse?" ( $0=$ none of my friends, $5=$ all of my friends). Higher scores indicated that adolescents perceived more friends as having sexual experiences.

To measure injunctive norms, adolescents' perceptions of their friends' (dis)approval toward sex were assessed at T2 with an adapted version of an item that has previously been used to measure parental sexual attitudes (Dittus \& Jaccard, 2000; East et al., 2006; Hill et al., 2018; Jaccard, Dittus, \& Gordon, 1996): "My best friends believe that boys and girls our age should not yet have sex" $(0=$ completely not true, 5 = completely true). Scores were reversed so that a higher score indicated that adolescents perceived that their friends were more approving of having sex.
Finally, to measure peer pressure, adolescents' experienced pressure from peers to have sex was assessed at T2 with one item from the Peer Pressure Scale (Santor et al., 2000): "I feel pressured to have sex, because many people my age have already had sex" ( $0=$ never, $5=$ very often) (East et al., 2006; Van de Bongardt et al., 2014, 2015). Higher scores indicated that adolescents experienced more pressure from their peers to have sex.

\section{Covariates}

In our analyses, we controlled for several $\mathrm{T} 1$ variables that are considered to be potential covariates of the investigated associations (Zimmer-Gembeck \& Helfand, 2008). In addition to adolescents' age, these covariates included: educational level, ethnic background, family structure.

\section{Educational Level}

Adolescents' educational level was assessed to reflect the various education levels in the stratified Dutch education system. About half of the adolescents in the present study sample (56.0\%) were enrolled in high educational levels (i.e., senior general education and pre-university education) and the other half $(44.0 \%)$ in low educational levels (i.e., the four types of pre-vocational education).

\section{Ethnic Background}

Adolescents' ethnic background was assessed by asking participating adolescents in which countries they themselves and their parents were born. The ethnic background variable was computed as consisting of two scores: $0=$ Dutch or another Western background, and $1=$ Non-Western background. The majority $(87.9 \%)$ of the participating adolescents in the present study had a Dutch or other Western ethnic backgrounds (i.e., adolescent and both parents born in the Netherlands, another country in Europe, or Australia), and $10.3 \%$ had a non-Western background (i.e., adolescent or at least one parent born in an African, Middle Eastern, Asian, or SouthAmerican country).

\section{Family Structure}

Adolescents' family structure was assessed by asking adolescents with whom they lived with most of the time $(0=$ Only with mother, 1 = With my mother and step-father, 2 = Only with my father, 3 = With my father and step-mother, $4=$ One half of the time with my mother and one half of the time with my father, 5 = Other, 6 = With my mother and my father). For the current analyses, the family structure variable was dichotomized $(0=$ Not living with both biological parents, which combined the response categories 0 through 5 and 1 = Living with both biological parents, which included response category 6).

\section{Data Analysis}

Descriptive statistics were used to portray the analysis sample characteristics. Chi-square tests and one-way ANOVA tests were performed in SPSS to assess gender differences in all variables. Mediation analyses were performed using Mplus version 7.4 to assess the direct associations between sexual 
communication with friends (online and face-to-face) at T1 and changes in adolescents' experience with sexual behaviors between T1-T3. Sexual peer norms at T2 (descriptive norms, injunctive norms, and peer pressure) were included as mediators. Figure 1 shows a schematic presentation of the tested mediation model. As sexual behavior was a count variable, and since a large proportion of our sample had a score of 0 (no previous sexual experience) on this variable, a zeroinflated Poisson distribution was specified for this variable in Mplus. According to Hilbe (2014), Poisson regression is an appropriate statistical method to apply when an outcome of interest represents count data with a large proportion of "zero" scores. We treated the mediators as continuous because they were measured using 6-point Likert-scales and corrected for the skewness of the mediators by using maximum likelihood estimation with robust standard errors (MLR) in Mplus.

Missing value analysis indicated that, in the final analysis sample, there were missing values among: family structure (5.7\%), sexual behaviors $(6.2 \%)$, and sexual communication with friends $(8.0 \%)$ at $\mathrm{T} 1$, descriptive norms $(16.8 \%)$, injunctive norms (11.6\%), and peer pressure $(13.2 \%)$ at $\mathrm{T} 2$, and sexual behaviors at T3 (11.3\%). To handle missing data in any of the variables in the model, we used the Full Information Maximum Likelihood (FIML). This method assumes missing at random (MAR) and is asymptotically equivalent to multiple imputations using all variables in the model, which is considered preferable over listwise deletion, even when values are not MCAR (Sterne et al., 2009).

The mediation analyses were conducted in three steps. Firstly, we fitted the hypothesized mediation model represented by paths: $c, a_{1}, a_{2}, a_{3}, b_{1}, b_{2}, b_{3}$, and c' (Figure 1) to the data. Secondly, we inspected and interpreted the estimates for the direct and indirect effects. To assess the total direct effect indicated by path $\mathrm{c}$, we examined the longitudinal association between online and face-to-face sexual communication with friends at T1 and changes in adolescents' sexual behaviors between T1-T3. To examine the direct effect indicated by path c', we assessed whether this association was explained by the three types of peer sexual norms (i.e., a set of mediators) at T2. We also calculated a total indirect effect to measure how much the three sexual peer norms together explained the association between sexual communication with friends at $\mathrm{T} 1$ and changes in sexual behaviors between T1-T3. This effect indicates how much of the observed reduction in the effect between sexual communication with friends and sexual behaviors, after the three mediators were added to the model, is due to the combined set of mediators (MacKinnon, 2008). After calculating the total indirect effect, we calculated the specific indirect effects for each mediator separately. With this, we assessed how much each particular sexual peer norm explained the main association, yet was conditional on the inclusion of the other two mediators in the model. Finally, we tested genderinteraction effects to examine whether there were statistically significant differences between boys and girls in the hypothesized associations. When significant gender-interaction effects were found, we performed the mediation analyses stratified by gender to show the paths separately for boys and girls. In all mediation models, we controlled for relevant covariates (i.e., gender, age, ethnic background, educational level, family structure), and for sexual behavior experience at $\mathrm{T} 1$ to assess change herein between $\mathrm{T} 1$ and T3. For all analyses, a significance level of $\mathrm{p}<.05$ was used to indicate significant effects.

\section{Results}

\section{Characteristics of the Study Sample}

The final prospective analysis sample included 1,116 adolescents (47.4\% girls) aged 11.5-17.9 years old across three waves (mean age at $\mathrm{T} 1=14.0$ years, $\mathrm{SD}=1.17$, and mean age at $\mathrm{T} 3=15.0$ years, $\mathrm{SD}=1.15)$. Table 1 shows that in the final prospective analysis sample, about $40.0 \%$ of adolescents had a low educational level, $87.0 \%$ were from a Dutch ethnic background, and $75.0 \%$ lived with both biological parents.

Table 1 also shows that, at T1, girls reported significantly more frequent online and face-to-face sexual communication with their friends than boys, $\mathrm{n}^{2}=.01$ (small effect size), $(p=<.001)$. At T2, perceptions of friends' sexual behaviors (descriptive norms) were similar between boys and girls $(p=.127)$. However, boys more often perceived that their

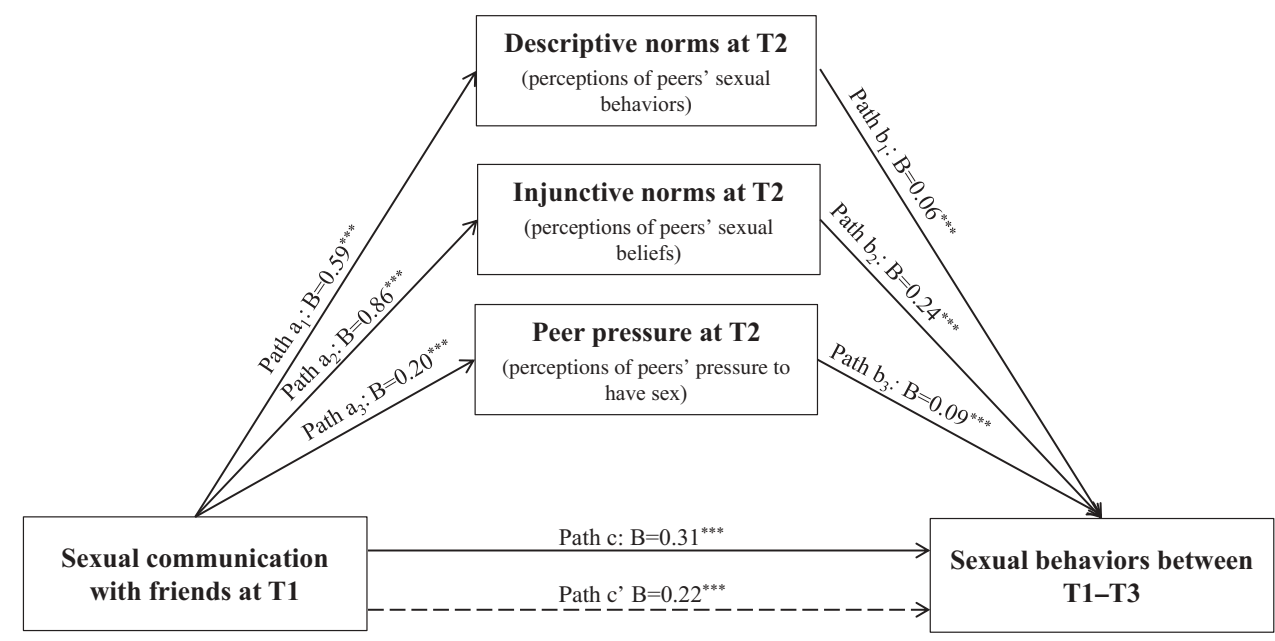

Figure 1. Tested mediation model. 
Table 1. Descriptive characteristics of the prospective analysis sample and gender differences.

\begin{tabular}{|c|c|c|c|c|c|c|c|c|}
\hline & \multicolumn{2}{|c|}{ Total $(n=1,116)$} & \multicolumn{2}{|c|}{ Boys $(n=587)$} & \multicolumn{2}{|c|}{ Girls $(n=529)$} & \multirow[b]{2}{*}{$p^{a}$} & \multirow[b]{2}{*}{ Cramer's $V^{b}$ or $n^{2 c}$} \\
\hline & $\mathrm{n}(\%)$ & Means (SD) & n (\%) & Means (SD) & $\mathrm{n}(\%)$ & Means (SD) & & \\
\hline \multicolumn{9}{|l|}{ T1 Covariates } \\
\hline Age & & & & & & & & .00 \\
\hline $11-17$ years & & $14.0(1.17)$ & & $14.0(1.20)$ & & $13.9(1.14)$ & .115 & \\
\hline Educational Level $^{d}$ & & & & & & & .002 & .09 \\
\hline Low & $442(40.0)$ & & $258(44.0)$ & & $184(34.8)$ & & & \\
\hline High & $674(60.0)$ & & $329(56.0)$ & & $345(65.2)$ & & & \\
\hline Ethnic Background & & & & & & & .128 & .05 \\
\hline Native Dutch & $967(87.0)$ & & $500(85.2)$ & & $467(88.3)$ & & & \\
\hline Non-native Dutch & $149(13.0)$ & & $87(14.8)$ & & $62(11.7)$ & & & \\
\hline Family Structure ${ }^{f}$ & & & & & & & .195 & .04 \\
\hline Living with both biological parents & $785(75.0)$ & & $405(73.0)$ & & $380(76.5)$ & & & \\
\hline Not living with both biological parents & $267(25.0)$ & & $150(27.0)$ & & $117(23.5)$ & & & \\
\hline Early Sexual Behaviors & & & & $0.3(0.92)$ & & $0.2(0.61)$ & .002 & .01 \\
\hline \multicolumn{9}{|l|}{ T1 Predictor } \\
\hline Sexual Communication with Friends & & $2.0(0.90)$ & & $1.9(0.88)$ & & $2.1(0.91)$ & $<.001$ & .01 \\
\hline \multicolumn{9}{|l|}{ T2 Mediators } \\
\hline Descriptive Norms & & $1.8(1.16)$ & & $1.8(1.20)$ & & $1.7(1.12)$ & .127 & .00 \\
\hline Injunctive Norms & & $3.3(1.81)$ & & $3.7(1.85)$ & & $2.3(1.70)$ & $<.001$ & .03 \\
\hline Peer Pressure & & $1.3(0.79)$ & & $1.4(0.93)$ & & $1.2(0.60)$ & $<.001$ & .02 \\
\hline \multicolumn{9}{|l|}{ T3 Outcome } \\
\hline Early Sexual Behaviors & & $0.5(1.15)$ & & $0.5(1.18)$ & & $0.4(1.12)$ & .412 & .00 \\
\hline
\end{tabular}

${ }^{a}$ Significance level of differences in characteristics measured at $\mathrm{T} 1$ between boys and girls by Chi-Square tests (categorical variables) and One-way ANOVA tests (continuous variables).

${ }^{\mathrm{b}}$ Cramer's V effect size, with $.01=$ small effect, .03 medium effect, .05 large effect (Rothman, Greenland, \& Lash, 2008)

${ }^{c} n^{2}=$ Eta-squared effect size, with $.01=$ small effect, $.06=$ medium, $.14=$ large effect (Rothman et al., 2008)

${ }^{d}$ Low educational level $=1=$ pre-vocational education. High educational level $=0=$ senior general education and pre-university education.

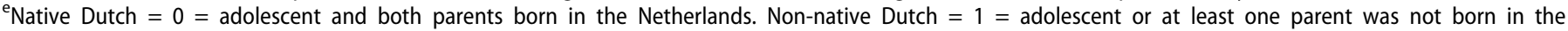
Netherlands.

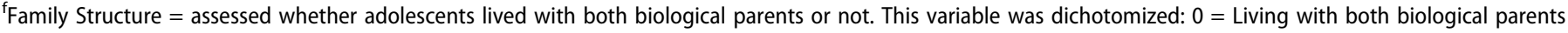
and $1=$ Not living with both biological parents.

friends approved more of having sex (injunctive norms) than girls, $\mathrm{n}^{2}=.03$ (small effect size), $(p<.001)$. Moreover, boys experienced significantly more pressure from their peers to have sex than girls, $\mathrm{n}^{2}=.02$ (small effect size), $(p<.001)$.

Regarding adolescents' experiences with different coital and noncoital sexual behaviors, our data show that, at T1, a total of 81 adolescents $(7.7 \%)$ were sexually experienced. Out of these, 51 (63.0\%) were boys, and 30 (37.0\%) were girls. Among these 81 adolescents who were sexually experienced at $\mathrm{T} 1$, the reported behaviors were: naked touching: 80 (99.0\%), manual sex: 81 (100\%), oral sex: 52 (64.2\%), and sexual intercourse: 50 (61.7\%).

At T3, a total of 147 adolescents (14.8\%) were sexually experienced, of which 80 (54.0\%) were boys, and 67 (46.0\%) were girls. Among these 147 adolescents who were sexually experienced at $\mathrm{T} 3$, the reported behaviors were: naked touching: 147 (100\%), manual sex: 143 (97.3\%), oral sex: 98 (67.0\%), and sexual intercourse: 92 (62.6\%). These prevalences were checked for consistency across the three waves.

\section{Tests of Mediation (Table 2; Figure 1)}

Figure 1 and Table 2 display the results of the mediation analysis that were performed to test the hypotheses.

\section{Direct Effect (Paths c) and Total Direct Effect (Path c')}

The findings from the tested mediation model (Figure 1) showed that the direct effect of sexual communication (online and face-to-face) on experience with sexual behaviors between $\mathrm{T} 1-\mathrm{T} 3$ is significant, $\mathrm{B}=0.31 ; p<.001$. That is, adolescents who communicated more frequently with their friends about sexuality-related topics at T1 reported significantly larger increases in their experience with sexual behaviors between T1-T3. When the three mediators were added in the model, the effect of sexual communication (online and face-to-face) on experience with sexual behaviors remained; unstandardized regression coefficient, $\mathrm{B}=0.22 ; p<.001$.

Table 2. Mediation analyses of the associations between online and face-to-face sexual communication with friends at $\mathrm{T} 1$ and adolescents' experiences with sexual behaviors between T1-T3, through perceived sexual peer norms at T2.

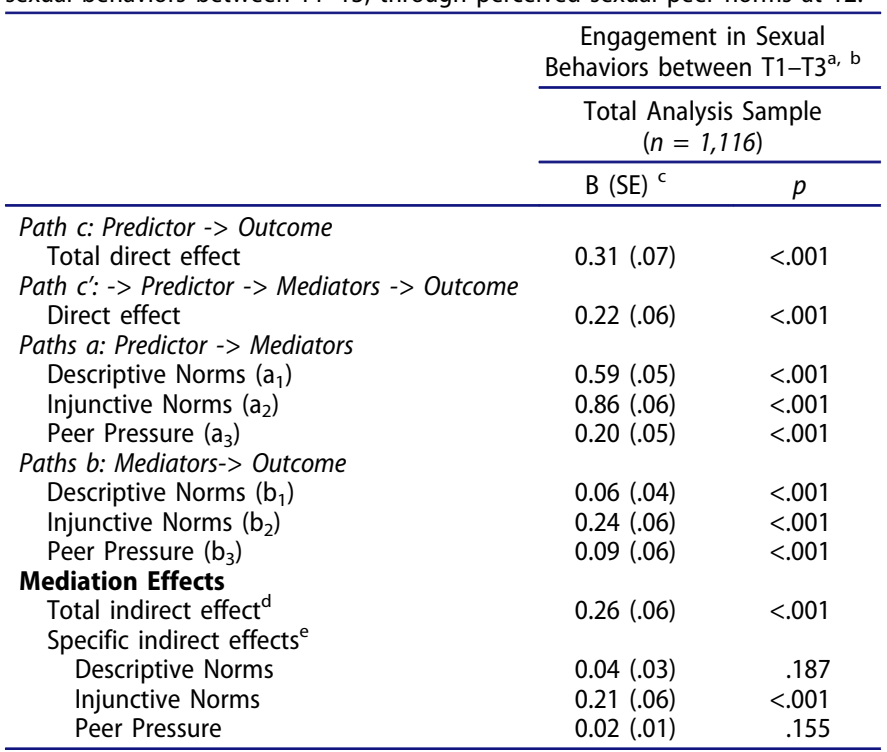

aAll analyses included covariates: gender, age (at T1), ethnic background, educational level (at T1), family structure (at T1), and sexual behaviors (at T1).

${ }^{b}$ No significant gender-interaction effects were found in any of the associations. ${ }^{\mathrm{C}} \mathrm{B}=$ unstandardized regression coefficients; $\mathrm{SE}=$ standard error.

${ }^{\mathrm{d}}$ Total indirect effect $=$ mediation effect of the three sexual peer norms together. ${ }^{\text {e}}$ Specific indirect effects = specific mediation effect of each sexual norm, yet conditional on the other two norms. 
Regarding gender differences in the tested mediation model, none of the paths was moderated by gender: $c(p=.631), \mathrm{a}_{1}(p=.059), \mathrm{a}_{2}(p=.631), \mathrm{a}_{3}(p=.728), \mathrm{b}_{1}$ $(p=.631), \mathrm{b}_{2}(p=.959), \mathrm{b}_{3}(p=.215)$. Thus, all hypothesized paths were similar between boys and girls.

\section{Adolescents' Sexual Communication with Friends at T1 and Three Sexual Peers Norms at T2 (Paths $a_{1}, a_{2}, a_{3}$ )}

The analyses for the total sample showed that more frequent online and face-to-face sexual communication with friends at T1 significantly predicted that 1 ) adolescents perceived more of their friends as having experience with sexual behaviors (descriptive peer norms) at $\mathrm{T} 2,(\mathrm{~B}=0.59 ; p<.001)$, and 2$)$ approving sexual behaviors at $\mathrm{T} 2(\mathrm{~B}=0.86 ; p<.001)$, and 3$)$ adolescents experienced more pressure from their peers to have sex $(\mathrm{B}=0.20 ; p<.001)$.

\section{Three Sexual Peer Norms at T2 and Experiences with Sexual Behaviors between T1-T3 (Paths $b_{1}, b_{2}, b_{3}$ )}

The analyses for the total analysis sample showed that adolescents who perceived more of their friends as 1) having sexual experiences (path $\left.b_{1}\right), 2$ ) approving of sex (path $b_{2}$ ), and 3) having more peer pressure (path $b_{3}$ ) at T2, reported significantly larger increases in experience with different sexual behaviors between T1-T3 (path $\mathrm{b}_{1}$ : $\mathrm{B}=0.06, p<.001$; path $\mathrm{b}_{2}: \mathrm{B}=0.24 ; p<.001$; path $\left.\mathrm{b}_{3}: \mathrm{B}=0.09 ; p<.001\right)$.

\section{Total Indirect Effect}

To test the combined mediating effect of the three sexual peer norms on the association between sexual communication with friends at $\mathrm{T} 1$ and changes in sexual behaviors between T1-T3, we calculated a total indirect effect, which was $\mathrm{B}=0.26$; $p<.001$.

\section{Specific Indirect Effects}

To test the individual mediating effects of the three sexual peer norms, yet conditional on the inclusion of the other two mediators in the model, we calculated the specific indirect effects (Table 2). The results for the total analysis sample showed that only the specific indirect effect of the injunctive norm was significant $(B=0.21 ; p<.001)$. This means that only injunctive norms mediated the association between sexual communication with friends at $\mathrm{T} 1$ and sexual behaviors between $\mathrm{T} 1-\mathrm{T} 3$.

\section{Discussion}

In the current study, we investigated the association between adolescents' sexual communication (online and face-to-face) with friends at $\mathrm{T} 1$ and changes in their experiences with sexual behaviors between T1-T3, examining whether this association was mediated by three types of sexual peer norms at T2 (i.e., descriptive, injunctive, and peer pressure). Moreover, gender differences were examined in all hypothesized associations.
In line with the previous literature (Busse et al., 2010), the first four hypotheses of our study were supported by our findings. H1: adolescents (both boys and girls) who had more frequent online and face-to-face sexual communication with their friends at $\mathrm{T} 1$ reported significant increases in experiences with sexual behaviors between T1-T3. H2: we found that adolescents who had more frequent sexual communication (online and face-to-face) with their friends at T1 significantly perceived, at $\mathrm{T} 2$, that: 1) more of their friends had experiences with sexual behaviors (descriptive norms), 2) their friends approved more of having sex (injunctive norms), and 3) there was more pressure from their peers to have sex (peer pressure). H3: our study showed that adolescents who, at T2, perceived that: 1) more of their friends had experiences with sexual behaviors, 2) their friends approved more of having sex, and 3) their friends exerted more pressure to have sex, had a significant increase in experiences with sexual behaviors between $\mathrm{T} 1-\mathrm{T} 3$. Regarding H4, our findings from the mediation analysis (i.e., total indirect effect) showed that the association between adolescents' online and face-to-face sexual communication with friends at T1 and sexual behaviors between T1-T3 was partially explained by the three sexual peer norms at T2, for both boys and girls. Moreover, when looking at the individual mediating effects of the three sexual peer norms, our results showed that only the specific indirect effect that mediated the main association was the injunctive norm. However, H5 was not supported by our findings as gender did not moderate any of the hypothesized associations.

The findings supporting $\mathrm{H} 1-4$ confirm the rationale that the mechanism of role modeling partly underlies these associations between sexual communication with friends and sexual peer norms (Bandura, 1971; Busse et al., 2010; Cialdini \& Goldstein, 2004). Specifically, it may be that during sexualityrelated talks, friends exchange information about their sexual behaviors, which may shape adolescents' perceptions of their friends' sexual behaviors, attitudes toward sex, and peer pressure to have sex. In turn, adolescents' perceptions of their friends' sexual peer norms could stimulate adolescents' sexual activity. That is, adolescents may reason that if their friends are having sex, it must be a good or wise thing to do the same (Bicchieri, 2017).

The role of injunctive norms in adolescents' increased experiences with sexual behaviors between $\mathrm{T} 1-\mathrm{T} 3$ may be related to the extent that these norms are in accordance with adolescents' values. When peers' approval of having sex is in line with adolescents' positive attitudes toward sexual behaviors, adolescents are more likely to initiate those behaviors themselves (Bicchieri, 2017; White, Hogg, \& Terry, 2002).

Moreover, they may be motivated to conform to their peers' behaviors (e.g., to engage in an expected behavior) because of perceived social benefits (e.g., social acceptance) when they do conform, or social losses (e.g., social rejection) when they do not conform (Bicchieri, 2017; Van de Bongardt et al., 2015). It has been shown that those adolescents who experienced more pressure from their peers to have sex were more likely to engage in sexual behaviors (Van de Bongardt et al., 2015). 
Our study contributes to advancing the understanding of how friends play a role in adolescents' sexual behaviors, through an interplay of communication and perceived norms about sex. However, our study showed that the three sexual peer norms together explained only part of the link between sexual communication with friends and sexual behaviors. That is, this link was partially mediated by the three sexual peer norms together, meaning that there are other factors that contribute to explain this link too (Doornwaard, Bickham, et al., 2014). An additional factor potentially affecting this link could be related to other (non-measured) aspects of the content discussed between adolescents and their friends, such as talks about when would be appropriate timing for initiating each sexual behavior (Doornwaard, Bickham, et al., 2014). The investigation hereof can be a direction for future studies. Moreover, it has been found that parents buffer the effects of peers on adolescents' engagement in sexual behaviors (Van de Bongardt et al., 2014). Indeed, a longitudinal study showed that more frequent sexual communication with parents significantly buffered the effects of perceiving more friends to be sexually active on adolescents' own intention to have sex (Van de Bongardt et al., 2014). Thus, even to understand how one social group, such as peers, may affect adolescents' sexual behaviors, we also need to take into account other factors like parents, and the content of sexual communication with friends.

Another possible reason underlying the link between adolescents' sexual communication with friends and their engagement in sexual behaviors between T1-T3 could be related to adolescents' personality traits. That is, some personality traits of adolescents could be related to both variables, sexual communication with friends, and sexual behaviors. Thus, adolescents who are more sociable, and adventurous than their peers, for example, could be more likely to talk to their friends about sexuality-related topics, and more likely to engage in sexual behaviors than their peers because of some aspects of their personality. These possible underlying mechanisms can also be directions for future studies in the field of adolescent sexual behavior.

Finally, not finding any gender differences in any of the hypothesized associations is in line with previous studies conducted in the Netherlands (Nogueira Avelar e Silva et al., 2016, 2018; Van de Bongardt et al., 2014). Perhaps, the culture of sexual double standards, in which boys and girls are generally incentivized to have different sexual behaviors by society is less expressive in this country, which might contribute to less evident gender differences in adolescents' sexual behaviors and socio-contextual correlates thereof (Kreager \& Staff, 2009).

\section{Strengths}

The current study had several strengths. Firstly, we used longitudinal data, which allowed us to assess the associations between adolescents' sexual communication with their friends and changes in their experience with different sexual behaviors through sexual peer norms over time. Thus, we were able to assess the predictive effect of sexual communication with friends at $\mathrm{T} 1$ on increases in sexual behaviors between
$\mathrm{T} 1$ and T3, and to what extent peers contribute to these modifications in sexual behaviors. Secondly, by measuring various sexual behaviors, ranging from naked touching to intercourse, and thus, not only intercourse, all adolescents who had experience with any of these behaviors were included in the present study. As such, the use of this broad conceptualization of sexual behavior allowed us to assess the role of peers in the initial stages of intimate and sexual behaviors in adolescence. Thirdly, our study also extended the literature by including both online and face-to-face communication measures. Considering the increasing contemporary popularity of online communication between adolescents and their peers (Doornwaard et al., 2015), the inclusion of online communication, in addition to face-to-face communication, is an evident strength of our study.

\section{Limitations and Future Directions}

The present study also had some limitations. Firstly, the generalizability of the results to other ethnic groups may be limited because our analysis sample consisted mainly of adolescents with a Dutch ethnic background. Secondly, the data were collected with adolescents' self-reports, which may have led to bias: It has been found that boys often over-report, and girls often underreport sexual experiences (Siegel, Aten, \& Roghmann, 1998). However, the longitudinal design of our study allowed us to check the over-time validity of adolescents' reports on their experience with sexual behaviors, after which we could correct or exclude inconsistencies in adolescents' reports over time. Thirdly, although in the present study we measured face-to-face and online sexual communication with friends, this measurement did not cover all possible means through which adolescents and their friends may talk about sexuality-related topics, such as different types of texting activity (e.g., WhatsApp), online chatting (e.g., Messenger), or social media (e.g., Facebook, Instagram, Twitter). Future studies should include means of communication that are popular among adolescents, in order to capture a broad contemporaneous reality (Collins et al., 2017; Doornwaard, Moreno, et al., 2014). Fourthly, although often used in studies on adolescent sexuality, one-item instruments may not fully grasp the complexity of peer influence processes. Multi-item scales, as well as more multi-method research (e.g., interviews, observations, experiments), are required to understand better the complex psycho-social processes that underlie the mechanisms of peer effects on adolescents' sexual behavior (Van de Bongardt et al., 2014). Furthermore, in our study, there were relatively low numbers of sexually experienced adolescents per type of sexual behavior, due to the relatively young age of the analysis sample (11.5-17.9 years). However, considering that we tested a complex model (with three mediators), for which we have also conducted complex moderated mediation analyses, running the analyses per sexual behavior was beyond the scope of this paper. This assessment could be a direction for future studies with larger analysis samples. Finally, research has shown that adolescents often have misperceptions of peers' risky behaviors (e.g., sexual behaviors), as they tend to overestimate the prevalence of such behaviors among their peers as well as peers positive attitudes toward the behaviors (Martens et al., 2006). These overestimations could also motivate adolescents toward becoming (more) sexually 
active. On the other hand, perceptions of peer behaviors and attitudes are stronger correlates of adolescents' own behaviors than actual peer-reported behaviors and attitudes, even when these perceptions are incorrect (Martens et al., 2006). Using peer education to inform adolescents about actual sexual behaviors and attitudes among youth (e.g., average ages at which most adolescents start engaging in various sexual behaviors) may be particularly effective for the correction of existing misperceptions, and the prevention of their effects on adolescents' own health behaviors (Agha \& Van Rossem, 2004; Caron, Godin, Otis, \& Lambert, 2004).

\section{Conclusions}

Overall, the findings of the current study-indicating that adolescents' perceptions of sexual norms among their peers partly explain the associations between how often they communicate about sex with their friends and increases in their experiences with different sexual behaviors over time-have implications for educators, health care professionals, and parents. When deploying prevention and intervention strategies to promote adolescents' sexual health, the influence of friends on adolescents' sexual behaviors through communication about sex and perceptions of sexual peer norms should be taken into account. Parents, teachers, and health care professionals should be aware of the possible role that peers may play a role in adolescents' decision making about their sexual behaviors. However, parents should also be aware that they play a relevant role in their children' experiences with sexual behaviors (Nogueira Avelar e Silva et al., 2016; Van de Bongardt et al., 2014).

\section{Acknowledgments}

We would like to thank the following statisticians for providing advice with regard to the statistical analyses: Joran Jongerling, Assistant Professor at the Department of Psychology, Erasmus University Rotterdam, the Netherlands; Stephen Kaptoge, Senior Statistician, Department of Public Health and Primary Care, University of Cambridge, UK; Daan Nieboer, and Lu Wang, researchers from the Department of Public Health, Erasmus Medical Center, the Netherlands.

\section{Disclosure statement}

No potential conflict of interest was reported by the authors.

\section{Financial Disclosure}

The authors have indicated they have no financial relationship relevant to this article to disclose.

\section{Funding}

Data for the current study were collected as part of a larger longitudinal study conducted in the Netherlands called "Project STARS" (Studies on Trajectories of Adolescent Relationships and Sexuality), which is funded by the Netherlands Organization for Scientific Research (NWO) and the Fund for Scientific Research on Sexuality (FWOS) (NWO Grant no. 43199-018). The Ph.D. project of the first author, of which the present study was a part, was supported by the Coordination for Improvement of Superior Level Personnel (CAPES) [Grant Number 7974/13-2].

\section{ORCID}

Raquel Nogueira Avelar e Silva (D) http://orcid.org/0000-0001-7895-0936

\section{References}

Agha, S., \& Van Rossem, R. (2004). Impact of a school-based peer sexual health intervention on normative beliefs, risk perceptions, and sexual behavior of Zambian adolescents. Journal of Adolescent Health, 34, 441-452. doi:10.1016/j.jadohealth.2003.07.016

Akers, A. Y., Gold, M. A., Bost, J. E., Adimora, A. A., Orr, D. P., \& Fortenberry, J. D. (2011). Variation in sexual behaviors in a cohort of adolescent females: The role of personal, perceived peer, and perceived family attitudes. Journal of Adolescent Health, 48, 87-93. doi:10.1016/j.jadohealth.2010.05.004

Ali, M. M., \& Dwyer, D. S. (2011). Estimating peer effects in sexual behavior among adolescents. Journal of Adolescence, 34, 183-190. doi:10.1016/j.adolescence.2009.12.008

Bandura, A. (1971). Social learning theory. New York, NY: General Learning Press.

Berten, H., \& Van Rossem, R. (2011). Mechanisms of peer influence among adolescents: Cohesion versus structural equivalence. Sociological Perspectives, 54, 183-204. doi:10.1525/sop.2011.54.2.183

Bicchieri, C. (2006). The grammar of society: The nature and dynamics of social norms. New York, NY: Cambridge University Press.

Bicchieri, C. (2017). Norms in the wild: How to diagnose, measure, and change social norms. New York, NY: Oxford University Press.

Bronfenbrenner, U. (1994). International encyclopedia of education (2nd edn). Oxford: Elsevier.

Busse, P., Fishbein, M., Bleakley, A., \& Hennessy, M. (2010). The role of communication with friends in sexual initiation. Communication Research, 37, 239-255. doi:10.1177/0093650209356393

Caron, F., Godin, G., Otis, J., \& Lambert, L. D. (2004). Evaluation of a theoretically based AIDS/STD peer education program on postponing sexual intercourse and on condom use among adolescents attending high school. Health Education Research, 19, 185-197. doi:10.1093/ her/cyg017

Cialdini, R. B., \& Goldstein, N. J. (2004). Social influence: Compliance and conformity. Annual Reviews, 55, 591-621. doi:10.1146/annurev. psych.55.090902.142015

Collins, R. L., Strasburger, V. C., Brown, J. D., Donnerstein, E., Lenhart, A., \& Ward, L. M. (2017). Sexual media and childhood well-being and health. Pediatrics, 140, S162-S166. doi:10.1542/ peds.2016-1758X

De Graaf, H., Van de Borne, M., \& Meijer, S. (2018). Offline and online sexual risk behavior among youth in the Netherlands: Findings from "Sex under the age of 25". Frontiers in Public Health, 1-10. doi:10.3389/fpubh.2018.00072

De Graaf, H., Vanwesenbeeck, I., Meijer, S., Woertman, L., \& Meeus, W. (2009). Sexual trajectories during adolescence: Relation to demographic characteristics and sexual risk. Archives of Sexual Behavior, 38, 276-282. doi:10.1007/s10508-007-9281-1

Dittus, P. J., \& Jaccard, J. (2000). Adolescents' perceptions of maternal disapproval of sex: Relationship to sexual outcomes. Journal of Adolescent Health, 26, 268-278. doi:10.1016/S1054-139X(99)00096-8

Doornwaard, S. M., Bickham, D. S., Rich, M., Vanwesenbeeck, I., Van de Eijnden, R. J. J. M., \& Ter Bogt, T. F. M. (2014). Sex-related online behaviors and adolescents' body and sexual self-perceptions. Pediatrics, 134, 1103-1110. doi:10.1542/peds.2014-0592

Doornwaard, S. M., Moreno, A. M., Van den Eijnden, R. J. J. M., Vanwesenbeeck, I., \& Ter Bogt, T. F. F. (2014). Young adolescents' sexual and romantic reference displays on Facebook. Journal of Adolescent Health, 55, 535-541. doi:10.1016/j.jadohealth.2014.04.002

Doornwaard, S. M., Van den Eijnden, R. J. J. M., Overbeek, G., \& Ter Bogt, T. F. M. (2015). Differential developmental profiles of adolescents using sexually explicit internet material. Journal of Sex Research, 52, 269-281. doi:10.1080/00224499.2013.866195

East, P. L., Khoo, S. T., \& Reyes, B. T. (2006). Risk and protective factors predictive of adolescent pregnancy: A longitudinal, prospective study. 
Applied Developmental Science, 10, 188-199. doi:10.1207/s1532480xads 1004_3

Fasula, A. M., \& Miller, K. S. (2006). African-American and Hispanic adolescents' intentions to delay first intercourse: Parental communication as a buffer for sexually active peers. Journal of Adolescent Health, 38, 193-200. doi:10.1016/j.jadohealth.2004.12.009

Hilbe, J. M. (2014). Modeling count data. New York, NY: Cambridge University Press.

Jaccard, J., Dittus, P. J., \& Gordon, V. V. (1996). Maternal correlates of adolescent sexual and contraceptive behavior. Family Planning Perspective, 28, 159-185. doi:10.1363/2815996

Kapungu, C. T., Baptiste, D., Holmbeck, G., Mcbride, C., RobinsonBrown, M., Sturdivant, A., ... Paikoff, R. (2010). Beyond the "birds and the bees": Gender differences in sex-related communication among urban African-American adolescents. Family Process, 49, 251-264. doi:10.1111/j.1545-5300.2010.01321.x

Kreager, D. A., \& Staff, J. (2009). The sexual double standard and adolescent peer acceptance. Social Psychology Quarterly, 72, 143-164. doi:10.1177/019027250907200205

MacKinnon, D. P. (2008). Introduction to statistical mediation analysis (1st ed.). New York, NY: Taylor \& Francis Group.

Martens, M. P., Page, J. C., Mowry, E. S., Damann, K. M., Taylor, K. K., \& Cimini, M. D. (2006). Differences between actual and perceived student norms: An examination of alcohol use, drug use, and sexual behavior. Journal of American College Health, 54, 295-300. doi:10.3200/JACH.54.5.295-300

Mollborn, S., \& Sennott, C. (2015). Bundles of norms about teen sex and pregnancy. Qualitative Health Research, 25, 1283-1299. doi:10.1016/j. alcr.2013.12.004

Nogueira Avelar E Silva, R., Van de Bongardt, D., Baams, L., \& Raat, H. (2018). Bidirectional associations between adolescents' sexual behaviors and psychological well-being. Journal of Adolescent Health, 62, 63-71. doi:10.1016/j.jadohealth.2017.08.008

Nogueira Avelar E Silva, R., Van de Bongardt, D., Van de Looij-jansen, P., Wijtzes, A., \& Raat, H. (2016). Mother- and father-adolescent relationships and early sexual intercourse. Pediatrics, 138, 1-9. doi:10.1542/peds.2016-0782

O'Sullivan, L. F., \& Brooks-Gunn, J. (2005). The timing of changes in girls' sexual cognitions and behaviors in early adolescence: A prospective, cohort study. Journal of Adolescent Health, 37, 211-219. doi:10.1016/j.jadohealth.2004.08.019

Reitz, E., Van de Bongardt, D., Baams, L., Doornwaard, S. M., Dalenberg, W., Dubas, J. S., \& Deković, M. (2015). Project STARS (Studies on Trajectories of Adolescent Relationships and Sexuality): A longitudinal, multi-domain study on sexual development of Dutch adolescents. European Journal of Developmental Psychology, 12, 613-623. doi:10.1080/17405629.2015.1018173

Rothman, J. K., Greenland, S., \& Lash, L. T. (2008). Modern epidemiology (3rd ed.). Philadelphia, PA: Wolters Kluwer, Lippincott Williams \& Wilkins.
Rudolph, K. D., \& Conley, C. S. (2005). The socioemotional costs and benefits of social-evaluative concerns: Do girls care too much? Journal of Personalized Medicine, 73, 115-138. doi:10.1111/j.1467-6494.2004.00306.x.

Santelli, J. S., Kaiser, J., Hirsch, L., Radosh, A., Simkin, L., \& Middlestadt, S. (2004). Initiation of sexual intercourse among middle school adolescents: The influence of psychosocial factors. Journal of Adolescent Health, 34, 200-208. doi:10.1016/j.jadohealth.2003.06.004

Santor, D. A., Messervey, D., \& Kusumakar, V. (2000). Measuring peer pressure, popularity, and conformity in adolescent boys and girls: Predicting school performance, sexual attitudes, and substance abuse. Journal of Youth and Adolescence, 29, 163-182. doi:10.1023/A:1005152515264

Sennott, C., \& Mollborn, S. (2011). College-bound teens' decisions about the transition to sex: Negotiating competing norms. Advances in Life Course Research, 16, 83-97. doi:10.1016/j.alcr.2011.05.001

Siegel, D. M., Aten, M. J., \& Roghmann, K. J. (1998). Self-reported honesty among middle and high school students responding to a sexual behavior questionnaire. Journal of Adolescent Health, 23, 20-28. doi:10.1016/S1054-139X(97)00274-7

Smetana, J. G., Campione-Barr, N., \& Metzger, A. (2006). Adolescent development in interpersonal and social contexts. Annual Review of Psychology, 57, 255-284. doi:10.1146/annurev.psych.57.102904.190124

Steinberg, L., \& Morris, A. S. (2001). Adolescent development. Annual Review of Psychology, 52, 83-110. doi:10.1146/annurev.psych.52.1.83

Sterne, J. A. C., White, I. R., Carling, J. B., Spratt, M., Royston, P., Kenward, M. G., Wood, A. M., \& Carpenter, J. R. (2009). Multiple imputation for missing data in epidemiological and clinical research: Potential and pitfalls. British Medical Journal, 338-393. doi:10.1136/bmj.b2393

Valkenburg, P. M., Sumter, S. R., \& Peter, J. (2011). Gender differences in online and offline self-disclosure in pre-adolescence and adolescence. British Journal of Developmental Psychology, 29, 253-269. doi:10.1348/2044-835X.002001

Van de Bongardt, D., De Graaf, H., Reitz, E., \& Deković, M. (2014). Parents as moderators of longitudinal associations between sexual peer norms and Dutch adolescents' sexual initiation and intention. Journal of Adolescent Health, 55, 388-393. doi:10.1016/j.jadohealth.2014.02.017

Van de Bongardt, D., Reitz, E., Sandfort, T., \& Deković, M. (2015). A meta-analysis of the relations between three types of peer norms and adolescent sexual behavior. Personality and Social Psychology Review, 19, 203-234. doi:10.1177/1088868314544223

Warner, T. D. (2018). Adolescent sexual risk taking: The distribution of youth behaviors and perceived peer attitudes across neighborhood contexts. Journal of Adolescent Health, 62, 226-233. doi:10.1016/j.jadohealth.2017

White, K. M., Hogg, M. A., \& Terry, D. J. (2002). Improving attitude-behavior correspondence through exposure to normative support from a salient in group. Basic and Applied Social Psychology, 24, 91-103. doi:10.1207/S15324834BASP2402_2

Zimmer-Gembeck, M. J., \& Helfand, M. (2008). Ten years of longitudinal research on U.S. adolescent sexual behavior: Developmental correlates of sexual intercourse, and the importance of age, gender, and ethnic background. Developmental Review, 28, 153-224. doi:10.1016/j.dr.2007.06.001 